\title{
HCG: Is it the Best Choice for Ovulation Triggering?
}

\author{
Peter Humaidan ${ }^{1}$, Daniel Bodri ${ }^{2}$, Evangelos G. Papanikolaou ${ }^{3}$ and Shahar Kol ${ }^{*}, 4$ \\ ${ }^{I}$ The Fertility Clinic, Odense University Hospital (OUH), Denmark \\ ${ }^{2}$ Clinica EUGIN, Barcelona, Spain \\ ${ }^{3}$ Assisted Reproduction Unit, Aristotle University of Thessaloniki, Greece \\ ${ }^{4}$ Department of Obstetrics and Gynecology, IVF Unit, Rambam Health Care Campus, Haifa, Israel
}

\begin{abstract}
With the increasing use of gonadotropin releasing hormone antagonist protocols, it has become an option to trigger ovulation with gonadotropin releasing hormone agonist which has several advantages over human chorionic gonadotropin triggering; among those an elimination or reduction in ovarian hyperstimulation syndrome, and a reduction in luteal phase discomfort. Thus, this paper questions the automated use of human chorionic gonadotropin for triggering of ovulation in assisted reproductive technologies.
\end{abstract}

Keywords: GnRH agonist, GnRH antagonist, hCG, in vitro fertilization, LH, OHSS.

\section{HUMAN CHORIONIC GONADOTROPIN VS. LH}

Human chorionic gonadotropin ( $\mathrm{hCG}$ ) has been the universal trigger of final oocyte maturation for decades in assisted reproductive technologies (ART) due to structural and biological similarities with LH as both molecules bind to the same receptor, the LH/hCG receptor [1]. Importantly, however, the half-life of hCG is significantly longer than that of and $\mathrm{LH},>24 \mathrm{~h}$ versus $60 \mathrm{~min}[2,3]$, facilitating ovarian hyperstimulation syndrome (OHSS) due to the sustained luteotropic activity and the production of vascular permeability mediators $[4,5]$. Moreover, even if bona-fide OHSS is not diagnosed, luteal ovarian over-stimulation is a significant source of physical discomfort during the luteal phase, increasing the treatment burden of the patient [6]. Interestingly, it has previously been reported that patients experience physical discomfort throughout the IVF treatment; however, even more discomfort is reported during the luteal phase compared with the stimulation phase [7]. Finally, a "standard" bolus of hCG has been shown to induce a significant histological advancement of the endometrium, which could negatively affect the receptivity [8]. This finding is in line with a recent prospective randomized trial, demonstrating the superiority of a spontaneous LH surge versus an hCG-induced surge in natural cycle frozen embryo transfer cycles. The authors hypothesized that the administration of hCG in the late follicular phase might negatively affect endometrial receptivity by inducing endometrial events which would occur several days later after an endogenous LH surge [9].

Taken together, the ovulatory bolus of hCG increases the physical discomfort of the patient during the luteal phase, increases the risk of OHSS and might hamper implantation.

*Address correspondence to this author at the Department of Obstetrics and Gynecology, IVF Unit, Rambam Health Care Campus, Haifa, Israel; Tel: +972-4-8300000; Fax: +972-4-8300093; E-mail: ivfisrael@gmail.com
The consented doses of hCG administered vary between 5,000 and 10,000 IU when urinary hCG is used and 6,500 IU (250 microgram) when recombinant hCG is used. Although hCG has been the drug of choice to trigger final oocyte maturation until now, we believe that the time has now come to question this practice.

The most natural compound with which to trigger final oocyte maturation and with an expected low incidence of OHSS would have been LH. Thus, when recombinant LH (rLH) was introduced, a clinical trial was performed to explore the efficacy of rLH to trigger ovulation in a long GnRHa down-regulation protocol. Doses differing between 5,000 IU and 30,000 IU were explored. However, apart from the significant increase in cost of treatment, a low implantation rate was seen after only one bolus of rLH to trigger ovulation; moreover, a disappointingly high OHSS rate $(12 \%)$ was reported $[10,11]$.

\section{GnRHa TRIGGERING IN ANTAGONIST ART CYCLES}

Following the introduction of GnRH antagonists for IVF/ICSI treatment, it became an option again to trigger final oocyte maturation with a GnRHa. This procedure has recently successfully been applied and is now the recommended protocol in oocyte donation cycles due to its many advantages: first and foremost a total elimination of OHSS in the donor, despite the retrieval of a considerable number of good quality mature oocytes, less discomfort in the "luteal phase" i.e. the days following oocyte aspiration, withdrawal bleeding within 4-6 days after triggering and finally and importantly, high pregnancy rates in the recipients [12-15].

In the initial randomized controlled IVF trials with transfer of the patients own embryos in a "fresh" cycle, however, the reproductive outcome was disappointingly low due to an unacceptably high early pregnancy loss rate [16, 17]. This finding was interpreted as an "insufficient luteal phase" after GnRHa triggering caused by low luteal LH 
levels. Following this study, a model for luteal phase rescue with exogenous LH activity administration (1500 IU hCG, significantly lower than the trigger dose) after GnRHa triggering was explored in a pilot study [18], reporting a comparable reproductive outcome after GnRHa triggering compared to hCG triggering. Subsequently the concept of luteal phase rescue with LH activity after GnRHa triggering has been confirmed in trials using either hCG or $\mathrm{rLH}$ for rescue [19-23]. A preliminary study [20] found a non-significant reduction of $7 \%$ in live birth rate with the use of GnRHa trigger compared to hCG. However, in a recent multicenter study including more than 100 patients in each group, this difference has disappeared, and we now see a non-significant higher ongoing pregnancy rate of $4 \%$ in favor of GnRHa trigger with the use of "tailored luteal phase support" (unpublished data). Thus GnRHa triggering is now a valid alternative to hCG triggering [24]. A Chochrane review by Youssef et al., [25] concluded that GnRH agonists as a final oocyte maturation trigger in fresh autologous cycles should not be routinely used due to the associated significantly lower live birth rate, ongoing pregnancy rate. However, the review included studies that differ significantly in the mode of luteal support, and therefore, is meaningless from the clinical perspective. A full account of this flawed and biased review was published by Humaidan et al. [26]

\section{GnRHa TRIGGERING AND FRIENDLY IVF PROTOCOLS}

"Friendly IVF" is the theme in recent years. Significant efforts have been made to make IVF a simple, "friendly" procedure. The medical industry identified the issue as a new market. An example is the long acting FSH, a product designed to reduce the number of injections during the stimulation phase, thereby alleviating the physical and emotional burden of the patient. Furthermore, clomiphenebased minimal stimulation or natural cycle IVF protocols in conjunction with GnRHa triggering have been proposed as valid alternatives to standard conventional IVF [27-29]. Interestingly, however, up till now, no commercial product or prospective trial targeted the luteal phase, which is a major source of patient discomfort.

Is it possible to implement a "friendly" luteal phase in ART? Yes, however, only if the hCG triggering property is dissociated from its luteal supportive property.

In this aspect, we believe that GnRHa triggering, followed by luteal LH activity support will be the future concept of triggering final oocyte maturation, significantly reducing or eliminating OHSS [12-14, 19, 30-33], significantly decreasing subjective luteal phase discomfort $[6,31]$ and increasing the number of MII oocytes [17, 20, 34, 35]. A recent "proof-of-concept" study explored the possibility of GnRHa trigger in IVF patients, with hCG-based luteal support [36]. The study suggests that two boluses of $1500 \mathrm{IU}$ hCG revert the luteolysis after a GnRHa trigger in the normo-responder patient. Importantly, no additional luteal support is needed. We therefore assume that progesteronebased luteal phase support (continuing well into the first trimester if pregnancy is achieved) will not be needed, once adequate LH-activity-based support is achieved (i.e. 2 hCG boluses). This will also decrease the overall cost of luteal support. This concept is further supported by Engmann et al., [37] who showed that granulosa/luteal cells obtained 2 days after GnRH agonist trigger are viable, and have the capacity to respond to hCG by increasing progesterone secretion.

In summary, GnRHa triggering is more patient friendly and offers several physiological advantages over hCG triggering. The time has now come to question the automated hCG triggering concept and move forward with thoughtful consideration of the needs and comfort of our patients.

\section{ACKNOWLEDGEMENT}

None declared.

\section{CONFLICT OF INTEREST}

None declared.

\section{REFERENCES}

[1] Kessler MJ, Reddy MS, Shah RH, Bahl OP. Structures of Nglycosidic carbohydrate units of human chorionic gonadotropin. J Biol Chem 1979; 254: 7901-8.

[2] Yen SS, Llerena O, Little B, Pearson OH. Disappearance rates of endogenous luteinizing hormone and chorionic gonadotropin in man. J Clin Endocr Metab 1968; 28: 1763-7.

[3] Damewood MD, Shen W, Zacur HA, Schlaff WD, Rock JA Wallach EE. Disappearance of exogenously administered human chorionic gonadotropin. Fertil Steril 1989; 52: 398-400.

[4] Papanikolaou EG, Pozzobon C, Kolibianakis EM, et al. Incidence and prediction of ovarian hyperstimulation syndrome in women undergoing gonadotropin-releasing hormone antagonist in vitro fertilization cycles. Fertil Steril 2006; 85: 112-20.

[5] Delvigne A, Rozenberg S. Epidemiology and prevention of ovarian hyperstimulation syndrome (OHSS): a review. Hum Reprod Update 2002; 28: 559-77.

[6] Cerrillo M, Rodriguez S, Mayoral M, Pacheco A, Martinez-Salazar J, Garcia-Velasco JA. Differential regulation of VEGF after final oocyte maturation with GnRH agonist versus hCG: a rationale for OHSS reduction. Fertil Steril 2009; 91: 1526-8.

[7] Boivin J, Takefman JE. Impact of the in-vitro fertilization process on emotional, physical and relational variables. Hum Reprod 1996; 11: 903-7.

[8] Fanchin R, Peltier E, Frydman R, de Ziegler D. Human chorionic gonadotropin: does it affect human endometrial morphology in vivo? Semin Reprod Med 2001; 19: 31-5.

[9] Fatemi HM, Kyrou D, Bourgain C, Van den Abbeel E, Griesinger G, Devroey P. Cryopreserved-thawed human embryo transfer: spontaneous natural cycle is superior to human chorionic gonadotropin-induced natural cycle. Fertil Steril 2010; 94: 2054-8.

[10] European Recombinant LH Study Group. Human recombinant luteinizing hormone is as effective as, but safer than, urinary human chorionic gonadotropin in inducing final follicular maturation and ovulation in in vitro fertilization procedures: results of a multicenter double-blind study. J Clin Endocr Metab 2001; 86: 2607-18.

[11] Aboulghar M, Al-Inany H. Triggering ovulation for IVF. Reprod Biomed Online 2005; 10: 142.

[12] Bodri D, Guillen JJ, Polo A, Trullenque M, Esteve C, Coll O. Complications related to ovarian stimulation and oocyte retrieval in 4052 oocyte donor cycles. Reprod Biomed Online 2008; 17: 237 43.

[13] Galindo A, Bodri D, Guillen JJ, Colodron M, Vernaeve V, Coll O. Triggering with HCG or GnRH agonist in GnRH antagonist treated oocyte donation cycles: a randomised clinical trial. Gynecol Endocrinol 2009; 25: 60-6.

[14] Hernandez ER, Gomez-Palomares JL, Ricciarelli E. No room for cancellation, coasting, or ovarian hyperstimulation syndrome in oocyte donation cycles. Fertil Steril 2009; 91: 1358-61.

[15] Melo M, Busso CE, Bellver J, et al. GnRH agonist versus recombinant $\mathrm{HCG}$ in an oocyte donation programme: a 
randomized, prospective, controlled, assessor-blind study. Reprod Biomed Online 2009; 19: 486-92.

[16] Kolibianakis EM, Schultze-Mosgau A, Schroer A, et al. A lower ongoing pregnancy rate can be expected when GnRH agonist is used for triggering final oocyte maturation instead of HCG in patients undergoing IVF with GnRH antagonists. Hum Reprod 2005; 20: 2887-92.

[17] Humaidan P, Bredkjaer HE, Bungum L, et al. GnRH agonist (buserelin) or hCG for ovulation induction in $\mathrm{GnRH}$ antagonist IVF/ICSI cycles: a prospective randomized study. Hum Reprod 2005; 20: 1213-20.

[18] Humaidan P, Bungum L, Bungum M, Yding Andersen C. Rescue of corpus luteum function with peri-ovulatory HCG supplementation in IVF/ICSI GnRH antagonist cycles in which ovulation was triggered with a GnRH agonist: a pilot study. Reprod Biomed Online 2006; 13: 173-8.

[19] Humaidan P. Luteal phase rescue in high-risk OHSS patients by GnRHa triggering in combination with low-dose HCG: a pilot study. Reprod Biomed Online 2009; 18: 630-4.

[20] Humaidan P, Ejdrup Bredkjaer H, Westergaard LG, Yding Andersen C. 1,500 IU human chorionic gonadotropin administered at oocyte retrieval rescues the luteal phase when gonadotropinreleasing hormone agonist is used for ovulation induction: a prospective, randomized, controlled study. Fertil Steril 2010; 93: 847-54.

[21] Castillo JC, Dolz M, Bienvenido E, Abad L, Casan EM, BonillaMusoles F. Cycles triggered with GnRH agonist: exploring lowdose HCG for luteal support. Reprod Biomed Online 2010; 20: 175-81.

[22] Humaidan P, Papanikolaou EG, Tarlatzis BC. GnRHa to trigger final oocyte maturation: a time to reconsider. Hum Reprod 2009; 24: 2389-94.

[23] Papanikolaou EG, Verpoest W, Fatemi H, Tarlatzis B, Devroey P, Tournaye H. A novel method of luteal supplementation with recombinant $\mathrm{LH}$, when a GnRH-Agonist is used instead of HCG for ovulation triggering. A randomized prospective proof of concept study. Fertil Steril 2011; 95: 1174-7.

[24] Kol S, Humaidan P. LH (as hCG) and FSH surges for final oocyte maturation: sometimes it takes two to tango? Reprod Biomed Online 2010; 21: 590-2.

[25] Youssef MAFM, Van der Veen F, Al-Inany HG, Griesinger G, Mochtar $\mathrm{MH}$, van Wely $\mathrm{M}$. Gonadotropin-releasing hormone agonist versus HCG for oocyte triggering in antagonist assisted reproductive technology cycles. Cochrane Database Syst Rev 2010; 11: CD008046.

[26] Humaidan P, Kol S, Engmann L, Benadiva C, Papanikolaou EG, Yding Andersen $\mathrm{C}$, on behalf of the Copenhagen GnRH Agonist
Triggering Workshop Group. Should Cochrane reviews be performed during the development of new concepts? Hum Reprod 2012; 27: 6-8.

[27] Zhang J, Chang L, Sone Y, Silber S. Minimal ovarian stimulation (mini-IVF) for IVF utilizing vitrification and cryopreserved embryo transfer. Reprod Biomed Online 2010; 21: 485-9.

[28] Matsuura T, Takehara Y, Kaijima H, Teramoto S, Kato O. Natural IVF cycles may be desirable for women with repeated failures by stimulated IVF cycles. J Assist Reprod Genet 2008; 25: 163-7.

[29] Teramoto S, Kato O. Minimal ovarian stimulation with clomiphene citrate: a large-scale retrospective study. Reprod Biomed Online 2007; 15: 134-48.

[30] Bodri D, Guillen JJ, Galindo A, Mataro D, Pujol A, Coll O. Triggering with human chorionic gonadotropin or a gonadotropinreleasing hormone agonist in gonadotropin-releasing hormone antagonist-treated oocyte donor cycles: findings of a large retrospective cohort study. Fertil Steril 2009; 91: 365-71.

[31] Engmann L, DiLuigi A, Schmidt D, Nulsen J, Maier D, Benadiva C. The use of gonadotropin-releasing hormone $(\mathrm{GnRH})$ agonist to induce oocyte maturation after cotreatment with $\mathrm{GnRH}$ antagonist in high-risk patients undergoing in vitro fertilization prevents the risk of ovarian hyperstimulation syndrome: a prospective randomized controlled study. Fertil Steril 2008; 89: 84-91.

[32] Kol S. Luteolysis induced by a gonadotropin-releasing hormone agonist is the key to prevention of ovarian hyperstimulation syndrome. Fertil Steril 2004; 81: 1-5.

[33] Manzanares MA, Gomez-Palomares JL, Ricciarelli E, Hernandez ER. Triggering ovulation with gonadotropin-releasing hormone agonist in in vitro fertilization patients with polycystic ovaries does not cause ovarian hyperstimulation syndrome despite very high estradiol levels. Fertil Steril 2010; 93: 1215-9.

[34] Imoedemhe DA, Sigue AB, Pacpaco EL, Olazo AB. Stimulation of endogenous surge of luteinizing hormone with gonadotropinreleasing hormone analog after ovarian stimulation for in vitro fertilization. Fertil Steril 1991; 55: 328-32.

[35] Oktay K, Turkcuoglu I, Rodriguez-Wallberg KA. GnRH agonist trigger for women with breast cancer undergoing fertility preservation by aromatase inhibitor/FSH stimulation. Reprod Biomed Online 2010; 20: 783-8.

[36] Kol S, Humaidan P, Itskovitz-Eldor J. GnRH agonist ovulation trigger and hCG-based, progesterone-free luteal support: a proof of concept study. Hum Reprod 2011; 26: 2874-7.

[37] Engmann L, Romak J, Nulsen J, Benadiva C, Peluso J. In vitro viability and secretory capacity of human luteinized granulosa cells after gonadotropin-releasing hormone agonist trigger of oocyte maturation. Fertil Steril 2011; 96: 198-202. 